\title{
Influencia del campo magnético sobre el crecimiento de microorganismos patógenos ambientales aislados en el Archivo Nacional de la República de Cuba
}

\author{
Matilde Anaya ${ }^{1}$, Eduardo Barbará2, Jesús Padrón², Sofía F. Borrego ${ }^{3}$, Oderlaise Valdés ${ }^{3}$, Alian Molina ${ }^{3}$ \\ 1 Instituto de Investigaciones para la Industria Alimentaria, La Habana, Cuba \\ 2 Departamento de Bioingeniería, Facultad de Ingeniería Eléctrica, Instituto Superior Politécnico José A. \\ Echeverría, La Habana, Cuba \\ ${ }^{3}$ Laboratorio de Conservación Preventiva, Archivo Nacional de la República de Cuba, La Habana, Cuba
}

Introducción. En el Archivo Nacional de la República de Cuba, existe contaminación electromagnética y la influencia del campo magnético oscilante de frecuencia extremadamente baja podría cuantificarse con microorganismos patógenos aislados de su ambiente interior.

Objetivo. Cuantificar la influencia de este tipo de campo magnético sobre el crecimiento de microorganismos patógenos aislados del ambiente en el Archivo Nacional de la República de Cuba.

Materiales y métodos. Se emplearon cinco microorganismos: Streptococcus sp. (1), Listeria sp. (2) y Candida guillermondii (3), aislados en el Archivo, así como Escherichia coli ATCC 25922 (4) y Saccharomyces cerevisiae (5), como referencia. Se les aplicó un campo magnético oscilante de frecuencia extremadamente baja de $60 \mathrm{~Hz} / 220 \mathrm{~V}$ de $3 \mathrm{mT}$ durante dos horas, en tres tubos de cultivo con agua destilada y con caldo nutriente. Después se inocularon $0,1 \mathrm{ml}$ en placas de Petri con los medios de cultivo agar CromoCen SC (1 y 2), agar de dextrosa y papa (3), agar CromoCen CC 4227 (4) y agar con extracto de malta (5). Las colonias se contaron (log UFC/ml) mediante el procesamiento digital de las imágenes de las placas de Petri empleando el programa MatLab ${ }^{\circledR}$.

Resultados. Se observó una estimulación significativa $(p \leq 0,05)$ de la cantidad de colonias tratadas con respecto a los controles, siendo mayor en el caldo nutriente que en el agua destilada y más en las bacterias (caldo nutriente-colonias tratadas: 9,43 a 10,62 UFC/ml) que en las levaduras (caldo nutriente-colonias tratadas: 8,31 a 8,79 UFC/ml). La estimulación se produjo en orden decreciente así: Listeria sp., E. coli ATCC 25922, Streptococcus sp., C. guillermondii y S. cerevisiae.

Conclusión. Se concluyó que el campo magnético aplicado tuvo un efecto estimulante sobre los microorganismos estudiados, lo cual potencia el riesgo para la salud del personal y los visitantes del Archivo Nacional de la República de Cuba.

Palabras clave: Streptococcus, Listeria, Candida, Escherichia coli, Saccharomyces cerevisiae, radiación no ionizante, virulencia, Cuba.

doi: http://dx.doi.org/10.7705/biomedica.v35i3.2569

Influence of magnetic field on the growth of pathogen microorganisms isolated from the indoor environment at the Archivo Nacional de la República de Cuba

Introduction: Electromagnetic pollution has been detected at the Archivo Nacional de la República de Cuba and the influence of extremely low frequency magnetic fields could be quantified with pathogenic microorganisms isolated from the indoor environment.

Objective: To quantify the influence of an extremely low frequency magnetic field on the growth of pathogenic microorganisms isolated from the environment at the Archivo Nacional.

Materials and methods: We used five microorganisms isolated at the Archivo Nacional: Streptococcus sp. (1), Listeria sp. (2) and Candida guillermondii (3), and Escherichia coli ATCC 25922 (4) and Saccharomyces cerevisiae (5) as references. We applied this magnetic field of extremely low frequency, $60 \mathrm{~Hz} / 220 \mathrm{~V}(3 \mathrm{mT})$, for two hours to these microorganisms on three culture tubes with distilled water

\footnotetext{
Contribución de los autores:

Matilde Anaya: diseño del estudio, ejecución de los aspectos prácticos, análisis e interpretación de resultados Eduardo Barbará: ejecución de los aspectos prácticos, análisis e interpretación de resultados Jesús Padrón: ejecución de los aspectos prácticos, recolección y análisis de resultados

Sofía Borrego: asesoramiento temático y metodológico en el diseño del estudio y análisis de resultados

Oderlaise Valdés: participación en el aislamiento de los microorganismos y ejecución de los aspectos prácticos

Alian Molina: identificación de los microorganismos y ejecución de los aspectos prácticos

Todos los autores participaron en la escritura del manuscrito.
} 
and nutrient broth. Then we inoculated $0.1 \mathrm{~mL}$ in the following solid culture mediums on Petri dishes: CromoCen SC Agar (1 and 2), Potato Dextrose Agar (3), CromoCen DC 4227 (4) and Malt Extract Agar (5). The colonies were counted (log $\mathrm{CFU} / \mathrm{mL}$ ) by digital processing of the images of Petri dishes using the MatLab ${ }^{\circledR}$ tool.

Results: We observed a statistically significant stimulation $(p \leq 0.05)$ in the quantity of treated colonies as compared to controls, which was higher in nutrient broth than in distilled water, and in bacteria (nutrient broth and treated colonies: 9.43 to $10.62 \mathrm{CFU} / \mathrm{mL}$ ) as compared with yeasts (nutrient brothtreated colonies: 8.31 to $8.79 \mathrm{CFU} / \mathrm{mL}$ ). In decreasing order, stimulation was as follows: Listeria sp., $E$. coli ATCC 25922, Streptococcus sp., C. guillermondii and S. cerevisiae.

Conclusion: We concluded that the magnetic field applied had a stimulating effect on the microorganisms under study, which increases the risk to the health of staff and visitors at the Archivo Nacional.

Key words: Streptococcus, Listeria, Candida, Escherichia coli, Saccharomyces cerevisiae, radiation, nonionizing; virulence, Cuba.

doi: http://dx.doi.org/10.7705/biomedica.v35i3.2569

El campo magnético (radiación no ionizante) puede provocar efectos dañinos o beneficiosos sobre los sistemas biológicos de todos los seres. El uso de dicha radiación como tratamiento magnético puede aplicarse en la modalidad de campo magnético estático u oscilante, es decir, un campo magnético que oscila según la frecuencia de la corriente eléctrica que lo induce (en $\mathrm{Hz}$ ). Las frecuencias menores de $3 \mathrm{kHz}$ se clasifican como frecuencias extremadamente bajas (1).

Los efectos del tratamiento magnético sobre los microorganismos se clasifican en no observables, inhibitorios o estimulantes, dependiendo de la densidad del campo magnético (B) expresada en teslas (T) o en gauss $(G)$, así como de la frecuencia de la corriente eléctrica que lo indujo, si es oscilante, y del tiempo de exposición (2). Para explicar dichos efectos se emplean varios mecanismos de forma individual o combinada, los cuales tienen en común el que actúan sobre las partículas cargadas y las moléculas de agua cuando se aplican en el medio de cultivo, y especialmente a nivel de membrana celular cuando inciden directamente sobre los sistemas biológicos (3).

En el caso de los efectos en los microorganismos, estos se relacionan no solo con su especie y su morfología $(4,5)$, sino también con las características del medio de cultivo (líquido o sólido) en el que reciben el tratamiento magnético (2). En ese sentido, se han estudiado más los efectos magnetobiológicos en medios de cultivo líquidos,

Correspondencia:

Eduardo Barbará, calle $114 \mathrm{~s} / \mathrm{n}$ e/ Ciclovía y Rotonda de la CUJAE, Marianao, La Habana, Cuba

Teléfono: (537) 2663713

eduardobm@electrica.cujae.edu.cu

Recibido: 07/10/14; aceptado: 25/03/15 utilizando técnicas indirectas como el recuento celular por densidad óptica o la cuantificación de un metabolito (6).

Para el caso del conteo de las colonias, las técnicas microbiológicas empleadas deben permitir la cuantificación de los microorganismos viables después de la exposición al tratamiento magnético aplicado, por ejemplo, la siembra en placas de Petri con medios de cultivo sólido adecuados. Deumeyer, et al. (7), demostraron que la concentración celular inicial es un factor importante para lograr estimular la fermentación alcohólica al aplicar campo magnético estático al cultivo celular de Saccharomyces cerevisiae, para lo cual estudiaron concentraciones celulares iniciales de entre $10^{2}$ y $10^{7}$ células $/ \mathrm{ml}$ en medio líquido con 2 y $6 \%$ de dextrosa, y concluyeron que $10^{3}$ células $/ \mathrm{ml}$ era la concentración inicial adecuada para obtener la mayor estimulación en este proceso, ya que con $10^{7}$ células $/ \mathrm{ml}$ los resultados no eran significativos (7).

No obstante, en los estudios con tratamiento magnético es difícil obtener modelos matemáticos que permitan predecir el grado de estimulación que manifiestan los microorganismos tratados magnéticamente para hacer la dilución correspondiente antes de inocularlos en las placas de Petri (3). Por lo tanto, el recuento de las colonias por esta vía puede dificultarse porque en ocasiones la cantidad es incontable y se incurre en errores. El procesamiento digital de imágenes es una herramienta útil para estudiar las variaciones que manifiestan los microorganismos $(8,9)$, por lo que podría utilizarse para cuantificar los efectos magnetobiológicos. Además, dicha herramienta puede aplicarse en sistemas automáticos para el análisis y el registro de colonias microbianas en placas de Petri (10). 
Por otra parte, se plantea que los efectos magnetobiológicos del campo magnético oscilante en frecuencias extremadamente bajas, de 50 o $60 \mathrm{~Hz}$, sobre los microorganismos, se deben a la genotoxicidad (cambios irreversibles del ADN) que este provoca $(11,12)$. Para estos valores de frecuencia se han informado resultados en un rango de densidad entre los $10 \mu \mathrm{T}$ y los $3 \mathrm{mT}$, lo cual puede deberse a factores como los géneros microbianos estudiados, el tiempo de exposición al tratamiento magnético y el medio de cultivo empleado.

En las investigaciones de Fojt, et al. $(4,5)$, con bacterias de diferente morfología, se concluyó que el bacilo Escherichia coli es más sensible que los cocos a un campo magnético oscilante en una frecuencia extremadamente baja de 50 $\mathrm{Hz}$ y $10 \mathrm{mT}$ durante 1 hora. Verschaeve, et al. (13), reportaron que con $50 \mathrm{~Hz}$ y densidades de 100 y $500 \mu \mathrm{T}$ durante 1 y 2 horas de exposición, no se pudo inducir genotoxicidad en un cultivo de Salmonella typhimurium, ni siquiera en combinación con otros agentes químicos mutágenos. Sin embargo, en un estudio reciente se planteó que con la exposición de un cultivo de $E$. coli a un campo de $3 \mathrm{mT}$ a $50 \mathrm{~Hz}$ durante 10, 14, 18 y 24 horas, se provocó la ruptura de la cadena de ADN del microorganismo (14).

Para el caso de las levaduras, la especie $S$. cerevisiae ha sido la más estudiada (15). Ager, et al. (16), informaron que no se habían podido inducir mutaciones en dicha especie empleando $1 \mathrm{mT}$ con $60 \mathrm{~Hz}$. Posteriormente, Motta (17) y Nakasono (18) informaron que la aplicación directa del tratamiento magnético sobre la célula no causó genotoxicidad. Las investigaciones de Gómez, et al. (19), demostraron que con densidades de 0,35 a 2,45 mT tampoco se produjeron cambios sustanciales en esta especie de levadura.

Ha podido observarse que, además de los factores de densidad y tiempo de exposición al campo magnético oscilante de frecuencia extremadamente baja, existen otros que amplían las condiciones experimentales de estas investigaciones: el género microbiano, la procedencia de la cepa, la morfología celular, la concentración celular inicial, el medio de crecimiento y su composición de nutrientes y el método de conteo celular. Esto evidencia la necesidad de llevar a cabo estudios que evalúen conjuntamente los factores mencionados.

En el semisótano del Archivo Nacional de la República de Cuba existen dos transformadores eléctricos que generan un campo magnético oscilante de $60 \mathrm{~Hz}$ de frecuencia. La presencia de microorganismos patógenos en esta área puede tener consecuencias para la salud de los trabajadores y de los visitantes de esta instalación, riesgo que se potenciaría con el estímulo al crecimiento de los microorganismos por efecto del aumento de la densidad de dicho campo magnético al acercarse a la fuente emisora.

Por todo lo planteado, el objetivo de este trabajo fue determinar la influencia del campo magnético oscilante de frecuencia extremadamente baja sobre el crecimiento de microorganismos patógenos aislados del ambiente en el Archivo Nacional, bajo diferentes condiciones experimentales y con el conteo de colonias mediante el procesamiento digital de imágenes.

\section{Materiales y métodos}

En la zona próxima al archivo fotográfico del Archivo Nacional, los valores de densidad del campo magnético oscilante de frecuencia extremadamente baja fluctúan entre 0,6 y 1,6 mT (lecturas hechas a cada metro a partir de la pared con un gaussímetro Enertech, Emdex II, USA).

Se evaluaron los siguientes factores experimentales: nutrientes en medios de cultivo (agua y caldo nutriente), tipo de microorganismos (bacterias y levaduras), medios sólidos de crecimiento selectivo (cromógenos y no cromógenos) y método de conteo (manual y automático), con aplicación de tratamiento magnético en un valor seleccionado y sin esta (cuadro 1).

\section{Caracterización de los microorganismos empleados en el estudio}

Se aislaron dos bacterias y una levadura del ambiente interior del archivo fotográfico. La levadura se identificó mediante pruebas bioquímicas $(20,21)$ y otras pruebas especializadas (formación de tubo germinativo, crecimiento a $37^{\circ} \mathrm{C}$ y prueba de hemólisis) en el Instituto de Medicina Tropical "Pedro Kouri" con un sistema de identificación de levaduras api42 ${ }^{\circledR}$ (BioMérieux2002, 20C AUX), y se confirmó que se trataba de Candida guillermondii (90 \% de confianza). Las dos bacterias Gram positivas se identificaron hasta el nivel de género como Listeria spp. y Streptococcus spp. mediante pruebas bioquímicas (22). Se les aplicó la prueba de hemólisis en agar nutriente con $5 \%$ de sangre de carnero sin fibrina y $0,1 \%$ de cloranfenicol, y se incubaron durante 24 a 48 horas a $37^{\circ} \mathrm{C}$, resultando ser cepas hemolíticas (datos no mostrados). 
Cuadro 1. Conteos de las colonias de microorganismos patógenos y de referencia

\begin{tabular}{|c|c|c|c|c|c|c|}
\hline \multirow{2}{*}{ Género microbiano } & \multirow{2}{*}{$\begin{array}{l}\text { Método de conteo } \\
\text { de colonias }\end{array}$} & \multirow{2}{*}{$\begin{array}{l}\text { Conteo de colonias } \\
\quad(\log \text { UFC/ml) }\end{array}$} & \multicolumn{2}{|c|}{ Agua destilada } & \multicolumn{2}{|c|}{ Caldo nutriente } \\
\hline & & & Control & TM & Control & TM \\
\hline \multirow[t]{4}{*}{ Candida guillermondii } & Manual & Mínimo & 7,92 & 8,16 & 8,33 & 8,78 \\
\hline & & Máximo & 7,96 & 8,19 & 8,34 & 8,79 \\
\hline & Automático & Mínimo & 7,83 & 8,00 & 8,30 & 8,78 \\
\hline & & Máximo & 7,95 & 8,17 & 8,33 & 8,79 \\
\hline Saccharomyces & Manual & Mínimo & 7,66 & 7,79 & 8,16 & 8,32 \\
\hline \multirow[t]{3}{*}{ cerevisiae } & & Máximo & 7,78 & 7,89 & 8,21 & 8,33 \\
\hline & Automático & Mínimo & 7,54 & 7,72 & 7,93 & 8,30 \\
\hline & & Máximo & 7,76 & 7,89 & 8,21 & 8,33 \\
\hline \multirow[t]{4}{*}{ Streptococcus spp. } & Manual & Mínimo & 8,30 & 8,78 & 8,90 & 9,43 \\
\hline & & Máximo & 8,60 & 8,95 & 9,04 & 9,51 \\
\hline & Automático & Mínimo & 8,30 & 8,78 & 8,90 & 9,43 \\
\hline & & Máximo & 8,85 & 8,95 & 9,04 & 9,51 \\
\hline \multirow[t]{4}{*}{ Listeria spp. } & Manual & Mínimo & 8,40 & 9,53 & 9,51 & 10,68 \\
\hline & & Máximo & 8,60 & 9,71 & 9,62 & 10,72 \\
\hline & Automático & Mínimo & 8,48 & 9,52 & 9,51 & 10,56 \\
\hline & & Máximo & 8,90 & 9,68 & 9,62 & 10,60 \\
\hline Escherichia coli & Manual & Mínimo & 8,48 & 8,90 & 8,90 & 9,64 \\
\hline \multirow{3}{*}{ ATCC 25922} & & Máximo & 8,90 & 9,32 & 9,08 & 9,81 \\
\hline & Automático & Mínimo & 8,48 & 8,90 & 8,90 & 9,64 \\
\hline & & Máximo & 8,90 & 9,32 & 9,08 & 9,81 \\
\hline
\end{tabular}

Nota: conteo manual de las colonias sobre placas de Petri de $90 \mathrm{~mm}$ llevado a cabo por técnicos especialistas y mediante conteo automático por procesamiento digital de las imágenes de las placas. TM: tratamiento con campo magnético oscilante de $3 \mathrm{mT}$ y $60 \mathrm{~Hz} / 220 \mathrm{~V}$ durante 2 horas, aplicado a cada género microbiano en tubos de cultivo con $5 \mathrm{ml}$ de agua destilada y de caldo nutriente

Se emplearon dos controles de referencia: $S$. cerevisiae, del banco de cepas del Instituto de Investigación para la Industria Alimentaria, y E. coli ATCC 25922, donada por la Facultad de Biología de la Universidad de La Habana.

\section{Preparación de las muestras}

Se utilizaron dos medios líquidos y dos concentraciones celulares iniciales diferentes, para evaluar el efecto del campo magnético oscilante de frecuencia extremadamente baja frente a la variación del contenido de nutrientes (6) y la densidad microbiana (7). Se prepararon tres tubos de ensayo con $5 \mathrm{ml}$ de agua destilada (medio de crecimiento líquido sin nutrientes y bajo contenido de sales) y otros tres con caldo nutriente (Biocen, Cuba), los cuales se inocularon con una concentración celular inicial ajustada a $10 \times 10^{6}$ y $10^{8}$ células $/ \mathrm{ml}$, y, posteriormente, recibieron el tratamiento magnético según se describe en la sección correspondiente y sin diluciones posteriores.

Para realizar el conteo de células viables después del tratamiento magnético, se tomaron $0,1 \mathrm{ml}$ de cada tubo y se inocularon en placas de Petri de $90 \mathrm{~mm}$ de diámetro con el medio de cultivo sólido (Biocen, Cuba) apropiado para cada género de microorganismo bajo estudio: agar de tripticasa de soya, agar McConkey, agar CromoCen SC y agar CromoCen CC 4227 para Listeria spp., Streptococcus spp. y E. coli ATCC 25922, respectivamente, y agar de dextrosa y papa y agar de extracto de malta para $C$. guillermondii y $S$. cerevisiae, respectivamente.

Todos los microorganismos se cultivaron por triplicado y se incubaron a $30{ }^{\circ} \mathrm{C}$ durante 24 a 48 horas según las normas ISO adoptadas en Cuba $(23,24)$, las cuales concuerdan con las normas de otros países $(25,26)$.

\section{Tratamiento magnético}

El tratamiento con el campo magnético oscilante de frecuencia extremadamente baja se hizo en un equipo experimental diseñado y elaborado por Tecnologías Electrónicas en Pinar del Río, Cuba, el cual fue avalado, calibrado y caracterizado por el Centro Nacional de Electromagnetismo Aplicado de Santiago de Cuba, Cuba. Este equipo genera una densidad de campo magnético (B) entre 0,5 y $120 \mathrm{mT}$ y consta de un generador de señal de corriente eléctrica de $60 \mathrm{~Hz} / 220 \mathrm{~V}$ y una bobina de cartón de tipo solenoide (con núcleo de aire) de $30 \mathrm{~cm}$ de diámetro y $29 \mathrm{~cm}$ de altura, con un alambre de cobre de $2 \mathrm{~mm}$ de diámetro, y una impedancia (resistencia a la corriente alterna) de $6,1 \Omega$. 
El tratamiento se aplicó con una densidad de $3 \mathrm{mT}$ (14) durante 2 horas bajo condiciones ajustadas a los resultados obtenidos en estudios similares $(27,28)$. Las muestras de control no recibieron tratamiento magnético.

\section{Captura de las imágenes}

Para capturar las imágenes se siguió la metodología empleada en estudios similares $(10,29)$. Se utilizó una cámara fotográfica digital marca Nikon apoyada sobre un soporte universal fijo a una distancia focal de $15 \mathrm{~cm}$, la cual captura imágenes RVA con resolución espacial de $3.648 \times 2.736$ píxeles de 8 bits cada uno. Se seleccionaron los medios de cultivo de mejor contraste para tomar las fotografías de las placas Petri. En total se procesaron 60 fotografías: cinco réplicas de los cuatro microorganismos cultivados por triplicado en el medio de cultivo de mejor contraste.

\section{Conteo de las células}

Para el desarrollo de la aplicación se utilizó el programa MatLab ${ }^{\circledR}$, versión 2013. Cada una de las imágenes pasó por tres etapas: lectura, procesamiento previo y conteo de las colonias (figura 1).

Después de llevar las imágenes a tonos de grises, se analizaron los histogramas para determinar los umbrales. El método de determinación de los umbrales utilizados en el proceso de segmentación, se basó en el análisis visual de los histogramas de las imágenes capturadas, es decir, mediante la inspección de estos valores en las imágenes. Se seleccionaron las regiones de interés con el método de manos libres y se segmentaron los objetos (colonias) por semilla para eliminar los bordes de las placas de Petri.

Por último, para el conteo de las colonias sobre las placas de Petri (variable de respuesta), se implementaron dos métodos independientes: el conteo de objetos binarios conectados (método 1) y la división de la cantidad de pixeles que ocupaban todas las colonias segmentadas entre la cantidad de píxeles promedio que representaba una sola colonia (método 2).

Además, el conteo de las colonias estuvo a cargo de dos técnicos especialistas ( $T$ ), cuyo resultado promedio se comparó con los obtenidos mediante el procesamiento digital de imágenes (PDI). Con la diferencia se determinó el porcentaje de error relativo de la operación (mediante el siguiente cálculo matemático: conteo T - conteo PDI / conteo T x 100), que no debía exceder el $52 \%$ permitido por las normas cubanas $(23,24)$, y se calculó el error relativo de los conteos obtenidos mediante la aplicación digital comparados con los promedios obtenidos por los técnicos especialistas.

A la cantidad de colonias resultante del conteo de los especialistas y la obtenida mediante el procesamiento digital de imágenes, se les aplicó el logaritmo decimal para efectuar el análisis estadístico. Los resultados debían variar según el

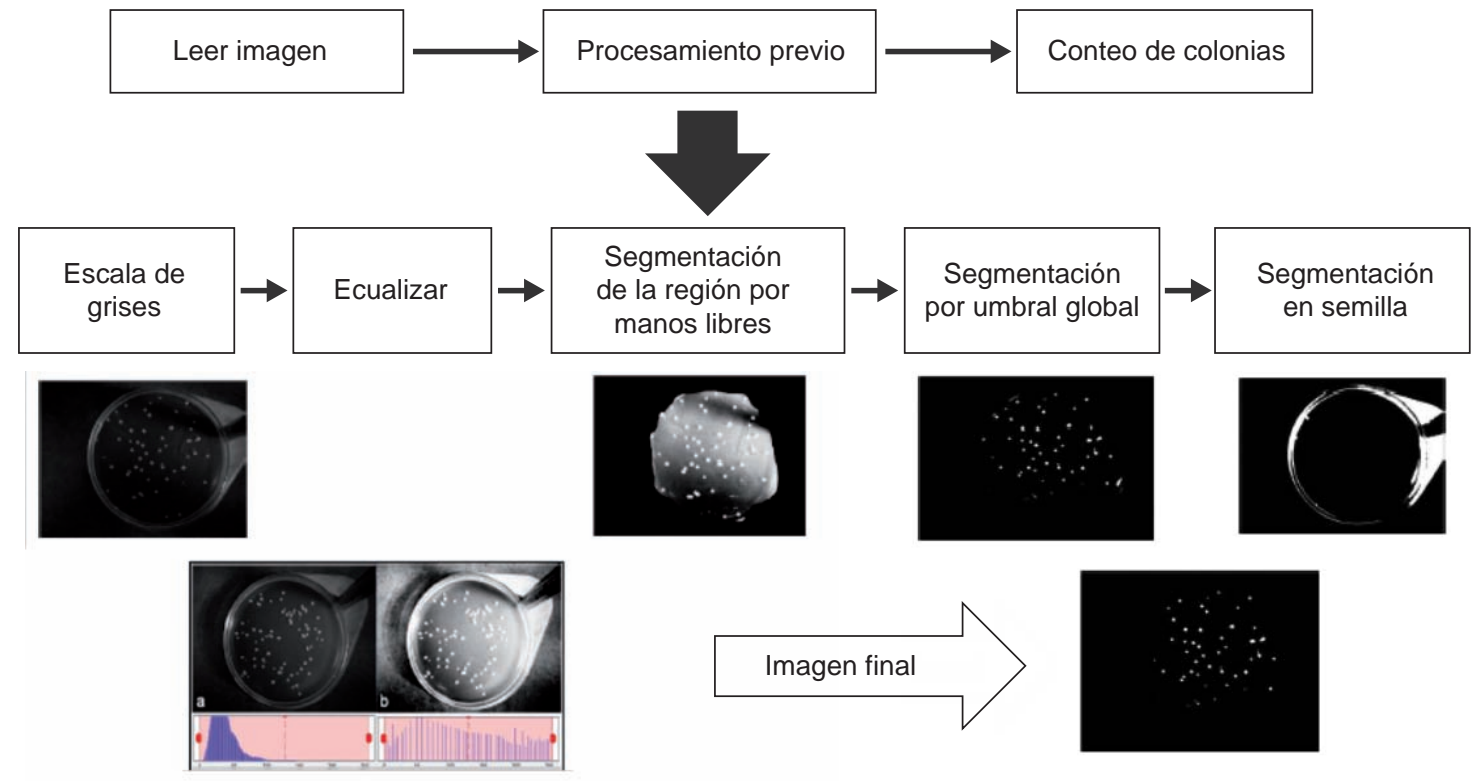

Figura 1. Diagrama de bloques del algoritmo del procesamiento digital de imágenes desarrollado para el conteo de las colonias microbianas 
valor exponencial de la concentración celular inicial correspondiente $\left(10^{6}\right.$ y $\left.10^{8}\right)$, por lo que el conteo de las colonias se expresó como logaritmo de las unidades formadoras de colonias por mililitro: log UFC/ml.

\section{Diámetro de las colonias}

Para su medición, se empleó el programa ImageJ 1.42, en el cual se introdujeron las imágenes, y de forma manual se marcó la distancia o el área del objeto deseado para que el programa arrojara el valor automáticamente (equivalencia de 0,2 $\mathrm{mm} /$ píxel).

\section{Análisis estadístico}

Se hicieron cinco réplicas de cada experimento y los resultados se procesaron con el programa Statgraphic Centurion XV. Se hizo un análisis de varianza simple (ANOVA) para determinar la diferencia del conteo de las colonias, según el medio de crecimiento líquido (agua destilada y caldo nutriente), el tratamiento magnético aplicado (cepas de control y cepas tratadas) y el grupo microbiano (bacterias y levaduras), aplicando rangos múltiples mediante el método de diferencia mínima significativa (DMS).

\section{Resultados}

\section{Análisis de las condiciones experimentales}

Después de ser tratados con el campo magnético oscilatorio de frecuencia extremadamente baja, todos los microorganismos seleccionados crecieron con su morfología típica (similar a la de los controles) en los medios de cultivo sólidos empleados para cada cepa, ya que no se observó en estos excreción de pigmentos, ácidos u otros metabolitos que alteraran su apariencia. Si las cepas sufrieron alguna modificación genotípica en los medios líquidos (agua destilada y caldo nutriente), no lo manifestaron en el crecimiento sobre los medios sólidos.

Sin embargo, en las placas de Petri con medios de cultivo sólidos hubo un contraste adecuado en las imágenes de los cultivos de las levaduras (figura 2a-d), pero no en los de Listeria spp. en agar de tripticasa de soya, debido a que este es un medio traslúcido de color similar a la colonia (figura $2 e$ y f), ni en el de $E$. coli en agar McConkey (figura $2 h$ ), debido al cambio de coloración que sufre este medio de cultivo como resultado del metabolismo celular de la cepa. Este comportamiento se observó con todas las bacterias, por lo que se seleccionó el medio CromoCen SC para Listeria

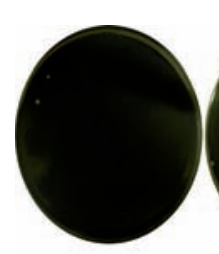

a

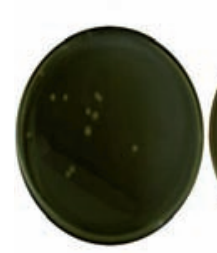

e

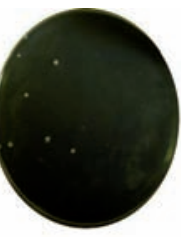

b

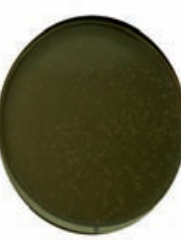

f

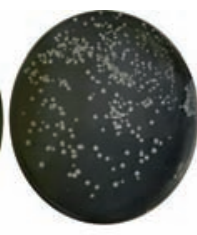

C

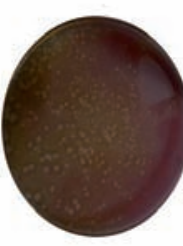

g

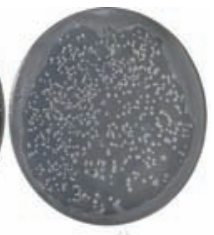

d

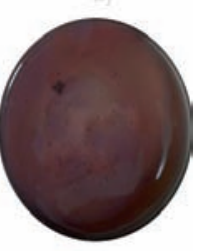

$\mathrm{h}$
Figura 2. Crecimiento de las colonias de levadura y de bacterias en placas de Petri después del tratamiento con el campo magnético oscilante de frecuencia extremadamente baja (60 Hz/220 V) de $3 \mathrm{mT}$ durante 2 horas (tiempo en el que las muestras de control se incubaron a $\left.30^{\circ} \mathrm{C}\right)$. S. cerevisiae tratada en agua destilada durante el crecimiento en agar de extracto de malta en diferentes concentraciones celulares (células/ml) ajustadas al inicio del experimento: a) $10^{8}$ células $/ \mathrm{ml}$, b) $10^{6}$ células $/ \mathrm{ml}$. En adelante la concentración inicial fue de $10 \times 10^{6}$ células/ml: $C$. guillermondii durante el crecimiento en agar de dextrosa y papa después de ser tratada en: c) agua destilada y d) caldo nutriente. Listeria sp. tratada en caldo nutriente: e) al inicio del experimento y durante el crecimiento en agar de tiripticasa de soya; tratada y durante el crecimiento en: f) agar de tripticasa de soya; g) en CromoCen SC; h) Escherichia coli ATCC 25922 empleada como control en agar McConkey

spp. y Streptococcus spp., mientras que para $E$. coli ATCC 25922 se empleó CromoCen CC 4227 (en dicho medio las otras dos bacterias fueron incoloras, lo que confirmó su identificación como Gram positivas después de iluminarlas con luz UV de $340 \mathrm{~nm}$ según el manual del fabricante).

En adelante se trabajó con dichos medios de cultivo y una concentración celular de $10^{8}$ y $10^{6}$ células $/ \mathrm{ml}$ para bacterias y levaduras, respectivamente, ya que no hubo diferencia estadísticamente significativa.

\section{Crecimiento de las colonias por efecto del campo magnético oscilante de frecuencia extremadamente baja}

El crecimiento de los cinco microorganismos estudiados resultó estimulado por el tratamiento magnético (figura 2), en tanto que, para un mismo medio de crecimiento líquido, el efecto del campo fue independiente de la concentración celular inicial analizada. Se hizo la segmentación automática de todas las imágenes obtenidas de las colonias en crecimiento en los medios de cultivo seleccionados. Algunos ejemplos de estas condiciones experimentales se muestran en la 
figura 3 para Streptococcus spp. y Listeria spp., en la figura 4, para E. coli ATCC 25922, y en la figura 5, para C. guillermondii y S. cerevisiae.

En las figuras 3, 4 y 5 puede observarse que el procedimiento empleado para el procesamiento digital de imágenes fue adecuado, demostrándose así la correcta selección del medio de cultivo sólido para lograr el contraste necesario en las imágenes sin afectar el metabolismo celular. Cuando el crecimiento fue elevado, el diámetro de las colonias de los bacilos (Listeria spp. y $E$. coli) fue dos veces mayor $(0,9 \pm 0,2 \mathrm{~mm})$ que el de la bacteria cocácea (Streptococcus sp., 0,6 $\pm 0,1 \mathrm{~mm}$ ). Esto facilitó el conteo visual hecho por el técnico, así como con los métodos de procesamiento digital de imágenes. En ese sentido, el conteo de las

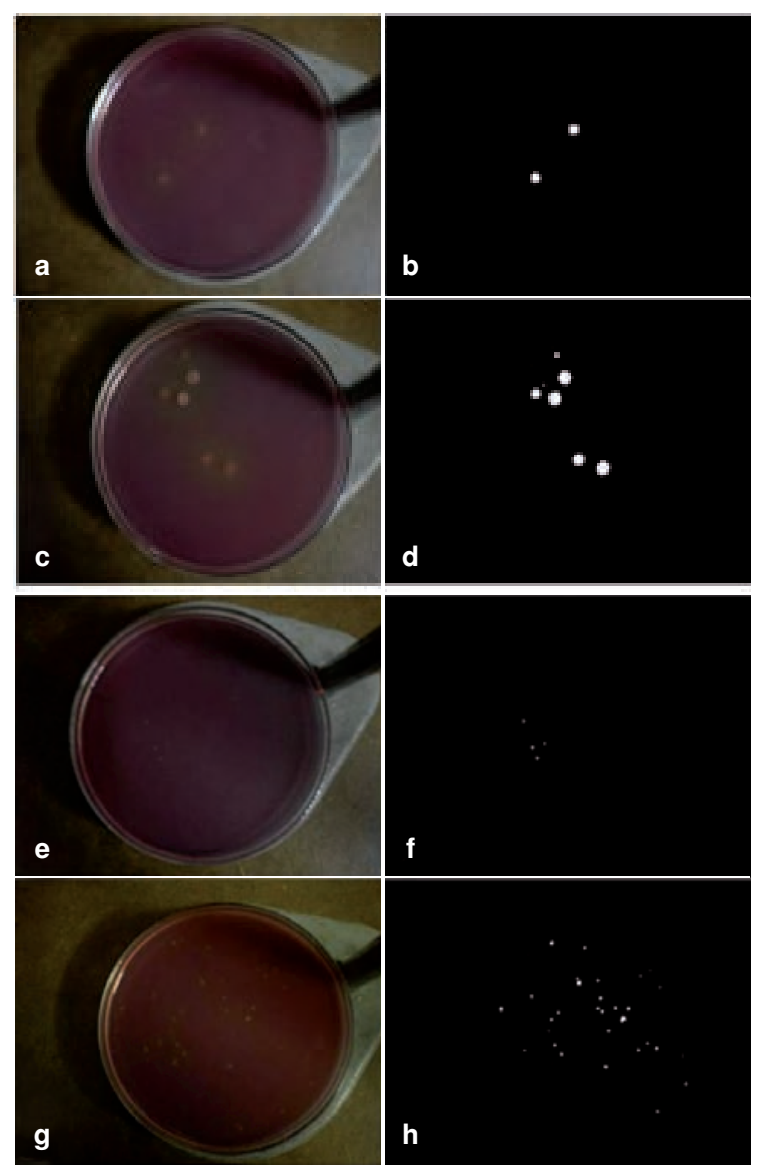

Figura 3. Crecimiento de colonias bacterianas en placas de Petri con medio CromoCen SC después del tratamiento con el campo magnético de frecuencia extremadamente baja (60 $\mathrm{Hz} / 220 \mathrm{~V}$ ) de $3 \mathrm{mT}$ durante 2 horas en tubos con agua destilada (concentración celular inicial de $10 \times 10^{8}$ células $/ \mathrm{ml}$; durante ese tiempo las muestras de control se incubaron a $30^{\circ} \mathrm{C}$ ). Imágenes originales y segmentadas de Streptococcus spp.: a y b) control; c y d) tratadas; y de Listeria sp.: e y f) control; g y h) tratadas.

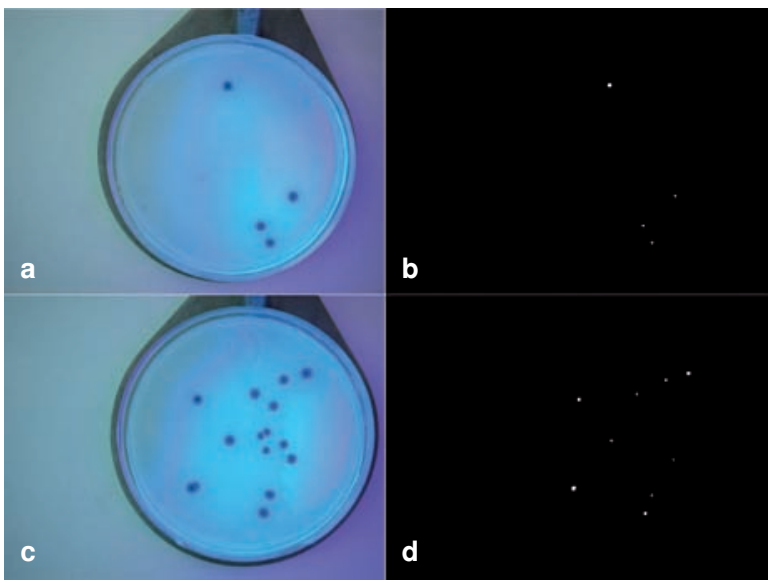

Figura 4. Crecimiento de las colonias de Escherichia coli ATCC 25922 en placas de Petri con medio CromoCen CC 4227 después de aplicar el tratamiento con el campo magnético de frecuencia extremadamente baja $(60 \mathrm{~Hz} / 220 \mathrm{~V})$ de $3 \mathrm{mT}$ durante 2 horas en tubos de cultivo con agua destilada (concentración celular inicial de $10 \times 10^{8}$ células $/ \mathrm{m}$; durante ese tiempo las muestras de control se incubaron a $30^{\circ} \mathrm{C}$ ). Imágenes originales iluminadas con luz UV de $340 \mathrm{~nm}$ y segmentadas: a y c: control; c y d: tratadas

colonias de levaduras fue mucho más práctico que el de la bacteria cocácea, ya que crecieron con mayor diámetro $(1,3 \pm 0,3 \mathrm{~mm})$.

\section{Conteo de las colonias mediante el procesamiento digital de imágenes}

El conteo de las colonias lo hicieron los técnicos y también se efectuó mediante el procesamiento digital de imágenes. En el cuadro 1 se muestran los resultados mínimos y máximos como indicativo de las concentraciones celulares obtenidas con tratamiento magnético y sin este. La mayor diferencia en los conteos en cada condición experimental se obtuvo en las levaduras y la mayor precisión se logró en E. coli ATCC 25922.

En todos los casos analizados se observó un aumento estadísticamente significativo $(p \leq 0,05)$ de la cantidad de colonias en las muestras tratadas con el campo magnético oscilante de frecuencia extremadamente baja comparada con la de los controles (figuras 6 y 7). El conteo de las colonias arrojó cantidades mayores en caldo nutriente que en agua destilada y en las bacterias (caldo nutriente-colonias tratadas: 9,43 a 10,62 UFC/ml) más que en las levaduras (caldo nutriente-colonias tratadas: 8,31 a 8,79 UFC/ml).

Para las bacterias se utilizó el método de conteo de objetos binarios conectados (método 1), ya que después de la segmentación y la conversión 

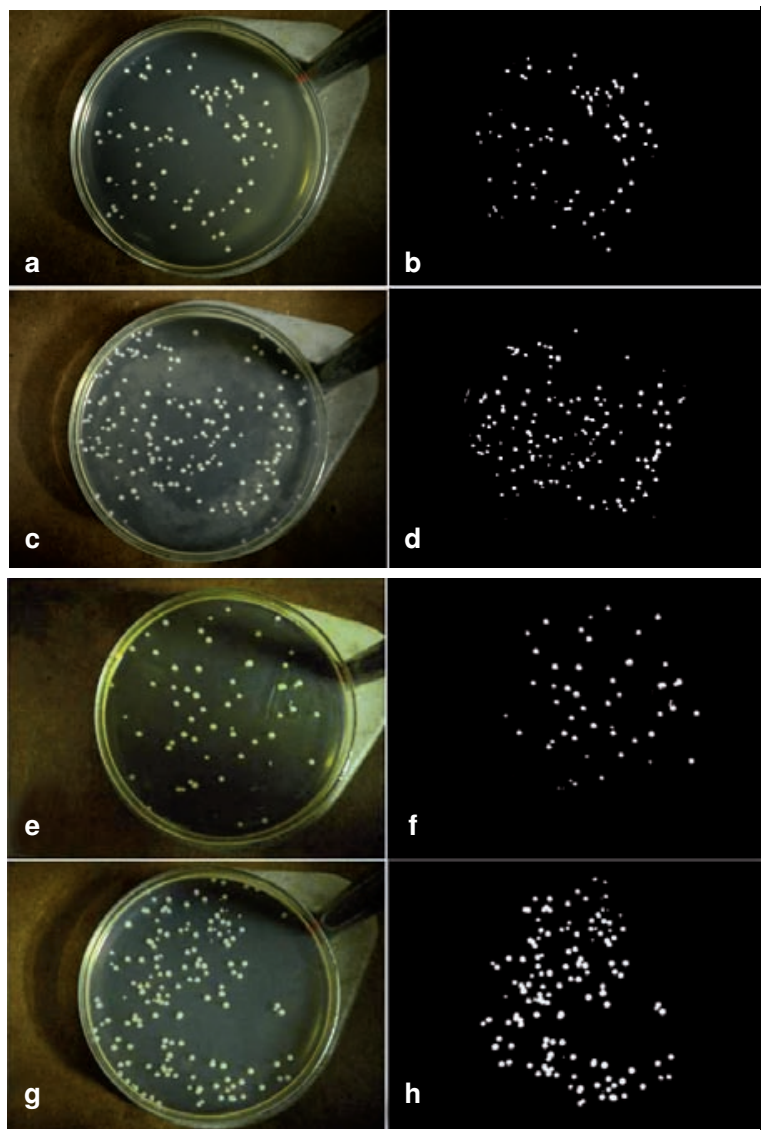

Figura 5. Crecimiento de las colonias de levaduras en placas de Petri después del tratamiento con el campo magnético de frecuencia extremadamente baja $(60 \mathrm{~Hz} / 220 \mathrm{~V})$ de $3 \mathrm{mT}$ durante 2 horas en tubos con agua destilada (concentración celular inicial de $10 \times 10^{6}$ células $/ \mathrm{ml}$; durante ese tiempo las muestras de control se incubaron a $30^{\circ} \mathrm{C}$ ). Imágenes originales y segmentadas: $C$. guillermondii en agar de dextrosa y papa: a y b) control; c y d) tratadas; $S$. cerevisiae en agar de extracto de malta: e y f) control; g y $h$ ) tratadas

a imágenes binarias, las colonias quedaron bien delimitadas. Solo hubo un error relativo en el conteo de las colonias de bacilos tratadas mediante el método 1 (5 a $27 \%$ ), porque durante la segmentación desaparecieron algunas colonias de diámetro pequeño $(0,6 \mathrm{~mm})$ que sí pudieron ser observadas por los técnicos especialistas. Esta situación no constituyó un problema, ya que el porcentaje de error se mantuvo en el orden numérico permitido (16 a $52 \%$ ) en las normas microbiológicas cubanas para alimentos $(23,24)$, y dado que en la práctica se observan variaciones entre los resultados obtenidos por diferentes técnicos especialistas.

De igual manera, se calculó el error relativo en las levaduras con cada uno de los dos métodos que se implementaron. Con el método 1 los errores relativos calculados estuvieron en el rango establecido en la norma citada. Sin embargo, se observó que, debido a los distintos diámetros, las colonias más pequeñas quedaban eliminadas cuando se erosionaban para obtener los objetos separados. Otra fuente de error del método fue el crecimiento de colonias unidas que, a pesar de la erosión, no se separaron y quedaron contadas como una sola colonia. Tampoco se contaron las colonias que crecieron unidas al borde de la placa después de la segregación, dado que compartían los mismos niveles de intensidad. Los errores con el método 2, al igual que con el método 1, se debieron fundamentalmente a las colonias que crecieron unidas al borde de la placa, por lo que fue mejor para el conteo de las colonias de levaduras que el método 1 (figura 7).

\section{Precisión del procesamiento digital de imágenes}

Con este método se logró una precisión correspondiente al orden de las dimensiones del píxel según la resolución de la cámara empleada en el proceso de captura de las imágenes. Es decir, no se pudieron medir las colonias menores al área que puede ocupar un píxel $\left(0,04 \mathrm{~mm}^{2}\right)$. La otra fuente de error que afectó la precisión del método se relacionó con las colonias de dimensiones menores al diámetro promedio $(0,2 \mathrm{~mm})$ que se vieron afectadas en el proceso de erosión morfológica binaria. Por último, también hubo colonias que no se contaron por haber crecido en el borde de la placa de Petri y que se eliminaron como resultado de la segmentación automática. Considerando estas tres fuentes fundamentales de error, los valores obtenidos con los métodos empleados se muestran en los cuadros 2 y 3 .

\section{Discusión}

Los resultados de la selección de las imágenes atendiendo al contraste entre el medio de cultivo y las colonias, son una evidencia de que este factor puede impedir al especialista su visualización. Además, la cantidad de colonias que llegan a crecer sobre una placa de Petri dificulta el conteo y puede propiciar el error.

Para el caso de las bacterias utilizadas en el trabajo, se empleó el método de conteo de objetos binarios conectados (método 1), ya que después de su segmentación y conversión a imágenes binarias, las colonias quedaron bien delimitadas, lo que favoreció su empleo. 


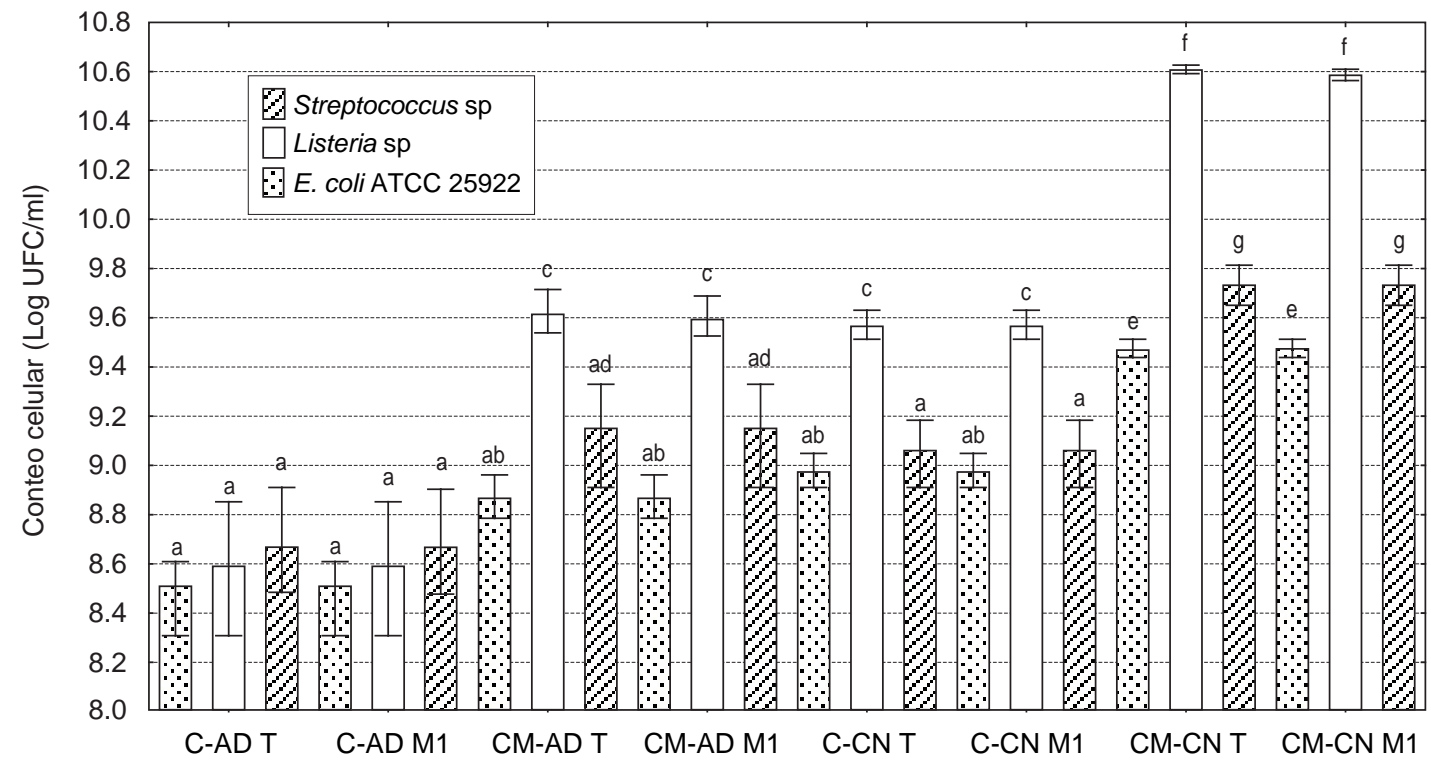

Figura 6. Cantidad de las colonias de bacterias en una placa de Petri de $90 \mathrm{~mm}$ expresada como logaritmo de las unidades formadoras de colonia por mililitro (log UFC/ml). Se inocularon $10 \times 10^{8}$ células $/ \mathrm{ml}$ en tubos de ensayo con $5 \mathrm{ml}$ de agua destilada (AD) y de caldo nutriente (CN) y se aplicó un campo magnético (CM) oscilante de $3 \mathrm{mT} / 60 \mathrm{~Hz}$ durante 2 horas (las muestras de control "C" no se sometieron al efecto del campo magnético). Se inocularon 0,1 ml en las placas de Petri y se incubaron durante 24 horas a $37^{\circ} \mathrm{C}$. Se contaron las colonias de dos formas: manual, a cargo de técnicos (T), y automática, por procesamiento digital de imágenes con conteo de objetos binarios conectados (M1). El promedio de n fue 15; las distintas letras indican una diferencia estadísticamente significativa $(p \leq 0,05)$ mediante el método de diferencia mínima significativa (DMS)

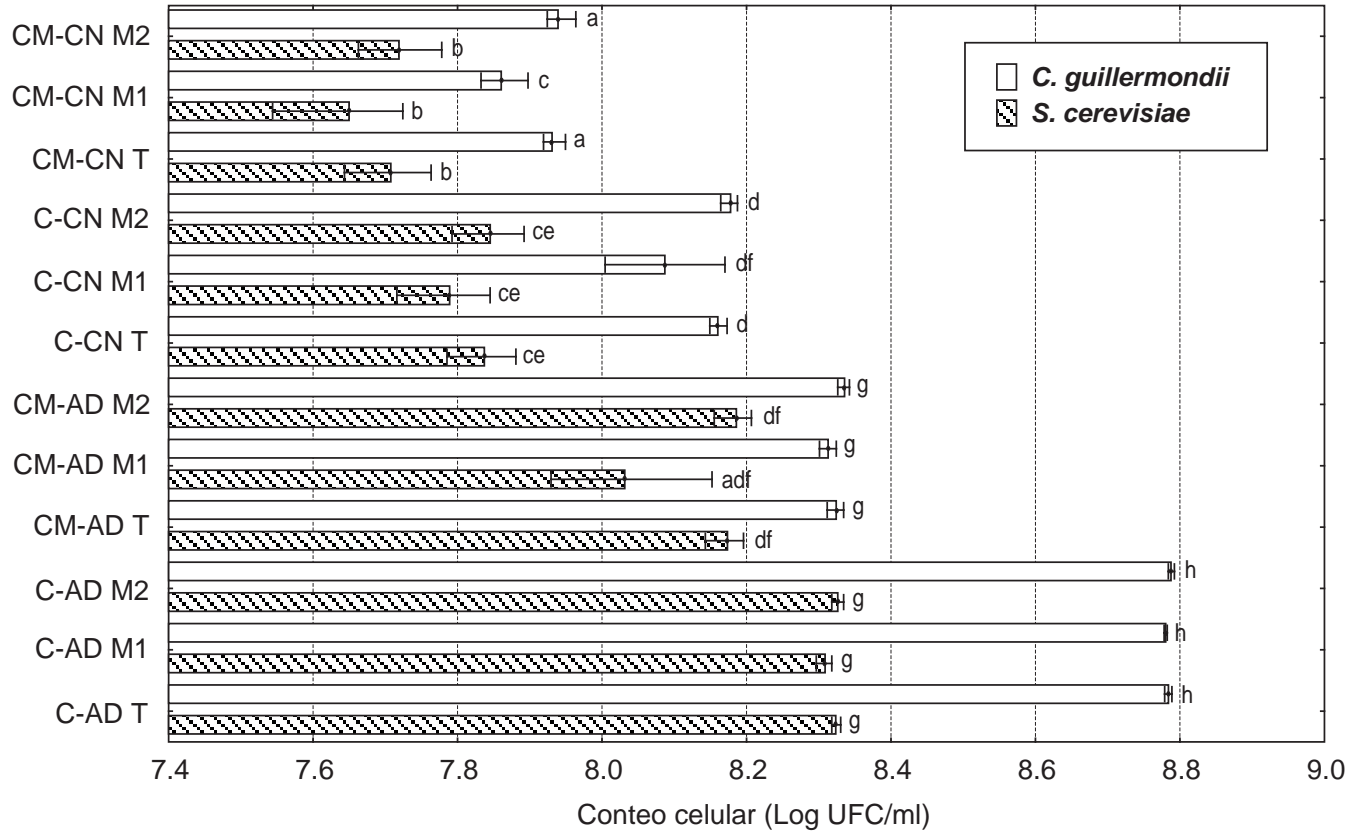

Figura 7. Cantidad de colonias de levaduras en una placa de Petri de $90 \mathrm{~mm}$ expresada como logaritmo de las unidades formadoras de colonia por mililitro (log UFC/ml). Se inocularon $10 \times 10^{6}$ células $/ \mathrm{ml}$ en tubos de ensayo con $5 \mathrm{ml}$ de agua destilada (AD) y de caldo nutriente $(\mathrm{CN})$ y se aplicó un campo magnético $(\mathrm{CM})$ oscilante de $3 \mathrm{mT} / 60 \mathrm{~Hz}$ durante 2 horas (las muestras de control "C" no se sometieron al efecto del campo magnético). Se inocularon $0,1 \mathrm{ml}$ en las placas de Petri y se incubaron durante 24 horas a $37 \stackrel{\circ}{\circ}$. Se contaron las colonias de tres formas: manual, a cargo de un técnico (T), automática, por procesamiento digital de imágenes con conteo de objetos binarios conectados (M1), y mediante la división de la cantidad de píxeles que ocupaban todas las colonias segmentadas entre la cantidad promedio de píxeles que representaban una sola colonia (M2). El promedio de $n$ fue 15; las letras distintas indican diferencias estadísticamente significativas $(p \leq 0,05)$ mediante el método de diferencia mínima significativa (DMS) 
Cuadro 2. Cálculo del error relativo cometido en la aplicación durante el conteo de las colonias de bacterias

\begin{tabular}{|c|c|c|c|c|}
\hline \multirow[t]{2}{*}{ Bacterias } & & \multicolumn{2}{|c|}{ Cantidad } & \multirow[t]{2}{*}{ Error relativo (\%) } \\
\hline & & Medida por el especialista & Calculada por la aplicación & \\
\hline \multirow[t]{2}{*}{ Streptococcus spp. } & Control & 2 & 2 & 0 \\
\hline & Tratada & 7 & 7 & 0 \\
\hline \multirow[t]{2}{*}{ Listeria spp. } & Control & 4 & 4 & 0 \\
\hline & Tratada & 40 & 38 & 5 \\
\hline \multirow[t]{2}{*}{ Escherichia coli ATCC } & Control & 4 & 4 & 0 \\
\hline & Tratada & 14 & 14 & 0 \\
\hline
\end{tabular}

Cuadro 3. Cálculo del error relativo cometido durante el conteo de las colonias de levaduras mediante los dos métodos

\begin{tabular}{lcccccc}
\hline Levaduras & & \multicolumn{3}{c}{ Cantidad } & & \multicolumn{2}{c}{ Error relativo (\%) } \\
\cline { 3 - 7 } & & $\begin{array}{c}\text { Medida por } \\
\text { especialistas }\end{array}$ & $\begin{array}{c}\text { Calculada por } \\
\text { el método 1 }\end{array}$ & $\begin{array}{c}\text { Calculada por } \\
\text { el método 2 }\end{array}$ & Método 1 & Método 2 \\
\hline C. guillermondii & Control & 87 & 72 & 85 & 17,20 & 2,29 \\
& Tratada & 150 & 125 & 145 & 16,60 & 3,30 \\
S. cerevisiae & Control & 53 & 44 & 45 & 16,90 & 15,00 \\
& Tratada & 148 & 108 & 152 & 27,00 & 2,70 \\
\hline
\end{tabular}

En el caso de las levaduras, se calculó de igual manera el error relativo ocurrido con cada uno de los dos métodos con respecto a la medición efectuada por los técnicos especialistas. Debe destacarse que los errores relativos (cuadros 2 y 3) están todos por debajo de los que plantean las normas de crecimiento microbiológico; por lo tanto, las aplicaciones desarrolladas mediante las técnicas de procesamiento digital de imágenes pueden emplearse satisfactoriamente.

En el análisis del conteo de colonias, Deutmeyer, et al. (7), explicaron varios de los factores por los que otros autores habían encontrado diferencias en los resultados concernientes a los efectos del campo magnético oscilante de frecuencia extremadamente baja sobre una misma especie microbiana. Entre los factores más importantes plantearon el origen de la cepa (cepas puras conservadas en laboratorios o cepas salvajes aisladas del ambiente) y la concentración celular inicial. En ese sentido, el hecho de que todos los microorganismos empleados en este estudio crecieran con su morfología típica en los diferentes medios de cultivo, evidenció que los efectos observados sobre el crecimiento celular fueron independientes de dichos factores.

La variabilidad de los medios de cultivo sólidos empleados posibilitó la apreciación a simple vista de la influencia del campo magnético sobre las colonias de los microorganismos, lo cual facilita su cuantificación por parte de los especialistas.
Sin embargo, se demostró que, para alcanzar mayor exactitud en el conteo de las colonias de las bacterias con el procesamiento digital de imágenes, es necesario emplear medios de cultivo cromogénicos.

En cuanto a la concentración celular inicial, los resultados de este estudio coinciden con los reportados por Deutmeyer, et al. (7), al tratar magnéticamente $S$. cerevisiae en una concentración celular de entre $10^{6}$ y $10^{7}$ células $/ \mathrm{ml}$, lo cual podría explicarse porque la estimulación del crecimiento celular en este rango de concentración provoca saturación de la densidad microbiana y agotamiento de los nutrientes en el medio líquido, lo que limita la cantidad de colonias en el medio sólido e impide posteriormente el aumento de su diámetro debido al mecanismo de autorregulación del crecimiento de los organismos unicelulares (30).

Con respecto al medio de crecimiento líquido, puede decirse que el efecto del campo magnético aplicado dependió del contenido de nutrientes en el medio. Este resultado concuerda con los observados por Morrow, et al. (6), al aplicar un campo electromagnético estático de 0,3 T sobre Streptococcus pyogenes en tampón fosfato salino y en caldo cerebro-corazón. Esto puede deberse a la estimulación de la actividad enzimática de las células, que les permite asimilar mejor los nutrientes, por el efecto del tratamiento magnético aplicado a las moléculas de agua y las partículas cargadas, fundamentalmente sobre los iones de 
calcio disueltos en el medio líquido. Al respecto, Brand, et al. (30), plantearon que el mecanismo de crecimiento de Candida albicans es dependiente del calcio, lo que pudiera explicar la mayor estimulación observada en $C$. guillermondii al tratarla magnéticamente en caldo nutriente que en agua destilada (figuras $2 \mathrm{~d}$ y $2 \mathrm{c}$, respectivamente).

Se observó que el efecto estimulante del campo magnético oscilante de frecuencia extremadamente baja aplicado sobre las bacterias, fue mayor en caldo nutriente que en agua destilada. Los bacilos tuvieron un mayor crecimiento que el coco tanto con tratamiento como sin este, siendo más significativo el de Listeria spp. que el de E. coli ATCC 25922 en agua destilada y caldo nutriente. Este resultado corrobora que los efectos magnetobiológicos sobre las bacterias dependen de su morfología celular $(4,5)$, lo cual determinará también su magnetorresistencia.

Al igual que con las bacterias, los efectos estimulantes del campo magnético aplicado sobre las levaduras fueron mayores en caldo nutriente que en agua destilada. Este resultado puede explicarse por su acción en las partículas cargadas (macromoléculas) del caldo nutriente, las cuales no están presentes en el agua destilada. Las cepas de levaduras tratadas incrementaron significativamente la cantidad de colonias comparada con las de control, siendo mayor la estimulación de $C$. guillermondii que la de $S$. cerevisiae.

Por lo tanto, los efectos magnetobiológicos se manifestaron en orden decreciente así: Listeria sp., E. coli ATCC 25922, Streptococcus sp., C. guillermondii y $S$. cerevisiae, lo que puede explicarse por la mayor velocidad de crecimiento y el menor tamaño de las células de las bacterias con respecto a las de las levaduras. Con base en este orden puede concluirse, además, que el campo magnético oscilante de frecuencia extremadamente baja aplicado tuvo mayor efecto sobre los microorganismos patógenos, ya que la levadura S. cerevisiae (de uso industrial en Cuba para la elaboración de cerveza), manifestó un aumento menor de la concentración celular.

Con los resultados de esta investigación se validó el empleo del procesamiento digital de imágenes como una herramienta para cuantificar el crecimiento de microorganismos. Con la metodología aplicada también pudo medirse el diámetro de las colonias que crecen sobre placas de Petri y se evidenció, además, la posibilidad de estimular el crecimiento de varios microorganismos en agua destilada o en otro medio líquido con escasos nutrientes exponiéndolos durante 2 horas a un campo magnético oscilante de frecuencia extremadamente baja de $3 \mathrm{mT}$ generado por fuentes emisoras de $60 \mathrm{~Hz}$ cercanas a los recipientes que las contenían. Este efecto magnetobiológico potencia el riesgo para la salud del personal y los visitantes del Archivo Nacional de la República de Cuba, y sirve de referencia para otros ambientes interiores. En estudios posteriores se podrá determinar si este factor físico en densidades más bajas provoca efectos similares.

\section{Agradecimientos}

A Carlos Fernández, del Departamento de Microbiología del Instituto de Medicina Tropical "Pedro Kouri", Cuba, por su ayuda en la identificación de la levadura Candida guillermondii aislada del ambiente en el Archivo Nacional de la República de Cuba.

\section{Conflicto de intereses}

Los autores declaramos que no existen conflictos de intereses para la publicación de este trabajo.

\section{Financiación}

Este estudio se llevó a cabo casi en su totalidad con el apoyo económico del Laboratorio de Conservación Preventiva del Archivo Nacional de la República de Cuba; aproximadamente un $10 \%$ de la financiación provino del proyecto 134/2010 del Programa de Ayuda para los Archivos de Iberoamérica (ADAI), España.

\section{Referencias}

1. Paunesku T, Woloschak GE. Effects of radiofrequency and extremely low-frequency electromagnetic field radiation on cells of the immune system. En: Barnes FS, Greenebaum B, editors. Biological and medical aspects of electromagnetic fields. Handbook of biological effects of electromagnetic fields. $3^{\text {th }}$ edition. Boca Raton, FL: Taylor \& Francis Group, LLC; 2007. p. 39-56.

2. Barbosa GV, Góngora MM, Swanson BG. Nonthermal electrical methods in food preservation. Food Sci Technol Int. 2000;4:363-70. http://dx.doi.org/10. $1177 / 108201329800400508$

3. Zapata JE, Moreno O, Márquez GF. Acción de un campo magnético sobre un cultivo aireado de Saccharomyces cerevisiae. Interciencia. 2005;30:40-6.

4. Fojt L, Klapetek P, Strašák L, Vetter V. $50 \mathrm{~Hz}$ magnetic field effect on the morphology of bacteria. Micron. 2009;40:91822. http://dx.doi.org/10.1016/j.micron.2009.06.009

5. Fojt L, Strasák L, Vetterl V, Smarda J. Comparison of the low-frequency magnetic field effects on bacteria Escherichia coli, Leclercia adecarboxylata and Staphylococcus aureus. Bioelectrochemistry. 2004;63:337-41. 
6. Morrow AC, Dunstan RH, King BV, Roberts TK. Metabolic effects of static magnetic fields on Streptococcus pyogenes. Bioelectromagnetics. 2007;28:439-45. http://dx.doi.org/10. 1002/bem.20332

7. Deutmeyer A, Raman R, Murphy P, Pandey S. Effect of magnetic field on the fermentation kinetics of Saccharomyces cerevisiae. Adv Biosci Biotechnol. 2011;2:207-13. http://dx. doi.org/10.4236/abb.2011.24031

8. Márquez JA, Sabino BA. Localización de células de la levadura Saccharomyces cerevisiae mediante procesamiento digital de imágenes. Revista Iberoamericana para la Investigación y el Desarrollo Educativo (RIDE). 2013;10:1-18.

9. Sabino BA, Márquez JA, Campos JM. Segmentación de células de la levadura Saccharomyces cerevisiae. Temas de Ciencia y Tecnología. 2011;15:3-8.

10. Osowsky J, Gamba HR. Sistema automático para contagem de colônias em placas de Petri. Rev Bras Eng Bioméd. 2001;17:131-9.

11. Hunt RW, Zavalin A, Bhatnagar A, Chinnasamy S, Das KC. Electromagnetic biostimulation of living cultures for biotechnology, biofuel and bioenergy applications. Int J Mol Sci. 2009;10:4515-58. http://dx.doi.org/10.3390/ ijms10104515.

12. Esmekaya MA, Acar SI, Kıran F, Canseven AG, Osmanagaoglu $O$, Seyhan N. Effects of ELF magnetic field in combination with iron (III) chloride $\left(\mathrm{FeCl}_{3}\right)$ on cellular growth and surface morphology of Escherichia coli (E. coli). Appl Biochem Biotech. 2013;169:2341-9. http://dx.doi. org/10.1007/s12010-013-0146-x

13. Verschaeve L, Anthonissen R, Grudniewska M, Wudarski J, Gevaert L, Maes A. Genotoxicity investigation of ELF-magnetic fields in Salmonella typhimurium with the sensitive SOS-based VITOTOX test. Bioelectromagnetics. 2011;32 580-4. http://dx.doi.org/10.1002/bem.20672

14. Gu S, Lu G, Wu Y, Li S, Zhao Y, Li K. A study of the interaction between ELF-EMF and bacteria. Adv Electric Electronics. 2012;155:243-54. http://dx.doi.org/10.1007/9783-642-28744-2_31

15. Binninger M, Ungvichian V. Effects of $60 \mathrm{~Hz}$ AC magnetic fields on gene expression following exposure over multiple cell generations using Saccharomyces cerevisiae. App Microbiol. 1996;20:140-53.

16. Ager D, Aradul J. Effect of $60 \mathrm{~Hz}$ magnetic fields on ultraviolet light-induced mutation and mitotic recombination in Saccharomyces cerevisiae. Mutat Res. 1992;283:279-86. http://dx.doi.org/10.1016/0165-7992(92)90060-U

17. Motta MA, Montenegro EJN, Stamford TLM, Silva AR, Silva FR. Changes in Saccharomyces cerevisiae development induced by magnet fields. Biotechnol Progr. 2001:970-3. http://dx.doi.org/10.1021/bp010076e

18. Nakasono S, Laramee C, Saiki H, McLeod K. Effect of power-frequency magnetic fields on genome-scale gene expression in Saccharomyces cerevisiae. Radiat Res. 2003;160:25-37.
19. Gómez MJ, Prieto MI, Ristori E, Martínez M. Static and 50 $\mathrm{Hz}$ magnetic fields of 0.35 and $2.45 \mathrm{mT}$ have no effect on the growth of Saccharomyces cerevisiae. Bioelectrochemistry. 2004;64:151-5.

20. De Hoog GS, Guarro J, Gene J, Figueras MJ. Yeasts and yeast-like fungi: Explanatory chapter, key to the genera and descriptions. Ascomycetous yeasts: Genus Candida. En: Atlas of clinical fungi. 2nd edition. Netherlands: Centraalbureau voor Schimmelcultures; 2000. p. 180-226.

21. Lodder J. Criteria and methods used in classifications of Candida. En: The yeast, a taxonomic study. Amsterdam: London Publishing Co; 1971. p. 34-113.

22. Bergy's manual of determinative bacteriology. Grampositive asporogenous rod-shaped bacteria. $8^{\text {th }}$ edition. Baltimore, USA: Williams \& Wilkins Co; 1975. p. 577-98.

23. Oficina Nacional de Normalización. Norma Cubana NC ISO 4833:2002. Microbiología de alimentos de consumo humano y animal. Guía general para la enumeración de microorganismos. Técnica de placa vertida a $30 \stackrel{\circ}{\circ}$. Ciudad de La Habana: Oficina Nacional de Normalización; 2002.

24. Oficina Nacional de Normalización. Norma Cubana NC ISO 4832:2002. Microbiología de alimentos de consumo humano y animal. Guía general para la enumeración de coliformes. Técnica de placa vertida a $30 \stackrel{\circ}{\circ}$. Ciudad de La Habana: Oficina Nacional de Normalización; 2002.

25. Estados Unidos Mexicanos - Secretaría de Salud. Norma Oficial Mexicana. NOM-092-SSA1-1994. Bienes y Servicios. Método para la cuenta de bacterias aerobias en placa. Ciudad de México, D.F.; Secretaría de Salud; 1995.

26. Swanson KM, Petran RL, Hanlin JH. Culture methods for enumeration of microorganisms. En: Downs F.P. \& Ito K (Editores); Compendium of methods for the microbiological examination of foods. 4th edition. Washington, D.C.: 2001. p. $53-67$.

27. Anaya M, Guzmán TG, Vivar A, Cobo HC, Acea CM. Comparación de la fermentación de dos cepas destileras $S$. cerevisiae en melaza tratada magnéticamente. Tsafiqui. 2013;4:21-7.

28. Guzmán TM, Anaya M, Mesa Y, Cobo HC. Células en cultivo de Lactobacillus acidophilus bajo la acción de un campo electromagnético de frecuencia extremadamente baja. Investigación y Saberes. 2013;2:13-7.

29. Farahani L, Etebarian H, Mohseni H, Sahebani N. Computer-based recognition of severity of apple blue mould using RGB components. International Research Journal of Applied and Basic Sciences. 2012;3:39-45.

30. Pirt SJ. A kinetic study of the mode of growth of surface colonies of bacteria and fungi. J Gen Microbiol. 1967;47:18197. http://dx.doi.org/10.1099/00221287-47-2-181

31. Brand A, Shanks S, Duncan VMS, Yang M, Mackenzie K, Gow NAR. Hyphal orientation of Candida albicans is regulated by a calcium-dependent mechanism. Curr Biol. 2007; 17:347-52. http://dx.doi.org/10.1016/j.cub.2006. 12.043 